\title{
Nanogels as nanocarriers for drug delivery: A review
}

\author{
Rahul Rajput, Jitendra Narkhede, Jitendra Naik* \\ University Institute of Chemical Technology, KBC North Maharashtra University, Jalgaon, 425001, India
}

*Corresponding Author: E-mail: jbnaik@nmu.ac.in

Received: September 16, 2019; Revised: December 12, 2019; Available online: December 21, 2019

\begin{abstract}
Nanogels are submicron-size aqueous dispersions of water-swollen particles, composed of nano-sized three-dimensional highly cross-linked networks of hydrophilic polymers. An active pharmaceutical agent or therapeutic agent with high or low molecular weight can be easily encapsulated into nanogels that can be delivered to the site of action via various routes, including oral, pulmonary, nasal, parenteral and intraocular routes, among others. Therapeutic agents encapsulated into nanogels improve the therapeutic activity in the biological environment. The application of different nanogels in drug delivery and recent clinical trial studies has been described concisely in this review.
\end{abstract}

(C)2020 by the authors. This article is an open-access article distributed under the terms and conditions of the Creative Commons Attribution license (http://creativecommons.org/licenses/by/4.0/).

\section{Keywords}

Drug; Polymers; Nanogels; Drug delivery; Applications

\section{Introduction}

Nanogels are commonly used in sensing, diagnostics, and bioengineering, but they are also often used in drug delivery [1,2]. Nanogels have benefits over conventional and macro-sized delivery systems because of their higher drug loading capacity, high stability, and improved contact time with the skin surface, which makes it more convenient as a transdermal drug delivery system. Water-soluble non-ionic polymers like hydroxylpropyl methylcellulose as well as ethylcellulose are commonly used to stabilise nanogel dispersions [3-7]. Phase separation of drug-loaded nanogels could occur due to interactions (electrostatic, hydrophobic, van der Waals) between the polymeric matrix and the active agent, which could be prevented by dispersing hydrophilic polymers. The dispersed hydrophilic polymer becomes exposed to the skin surface by forming a protective layer around the nanogel, allowing drug particles to remain dispersed in the gel matrix [8-10]. Modified natural biopolymers possess a high degree of functional groups with additional functional crosslinkers used for the formulation of biopolymer-based nanogels. Innovative techniques such as photopolymerisation, chemical cross-linking, click chemistry-based cross-linking etc., are used to derive the self-assembly and cross-linking of hydrophilic block copolymers. Between internal and external layers of nanogels, block polymers permit the control of drug release from a polymer matrix [11-13]. For targetspecific or cell-specific drug delivery, nanogels are modified with ligands to permit receptormediated drug release at the site of action [14-15]. Drug- or biologically-loaded nanogels cross biological barriers and release the therapeutic agent inside cells [16-18]. In recent years, nanogels were effectively 
utilised in the field of biotechnology to deal with genetics, enzyme immobilisation and protein synthesis, thus providing an efficient tool to cater for novel therapeutic systems in medicine (Table 1). A novel coreshell magnetic nanogel was prepared using poly(acrylamide) for cancer therapy. Pluronic poly(ethyleneimine) was used to prepare a thermoresponsive nanogel for the transdermal delivery of an active agent. Nanogel-based drug delivery formulations increase the effectiveness and safety of certain anti-cancer drugs as well as many other drugs due to their chemical composition, which has been confirmed by in vivo studies in animal models. Nanogels are a favourable and innovative drug delivery system that can play a vital role by addressing the problems associated with old and modern therapeutics such as nonspecific effects and poor stability [19-23].

Table 1. Applications and types of nanogel in drug delivery [21-23]

\begin{tabular}{|c|c|c|}
\hline Polymer & Type of Nanogel & Uses \\
\hline Pullulan/folate-pheophorbide & $\begin{array}{l}\text { Self-quenching } \\
\text { polysaccharide-based }\end{array}$ & Minimal phototoxicity of pheophorbide \\
\hline $\begin{array}{l}\text { Cross-linked branched network } \\
\text { of poly(ethyleneimine) and PEG }\end{array}$ & Polyplex nanogel & $\begin{array}{c}\text { Elevated activity and reduced cytotoxicity } \\
\text { of fludarabine }\end{array}$ \\
\hline Acetylated chondroitin sulphate & Self-organising nanogel & Doxorubicin loaded \\
\hline Heparin pluronic nanogel & Self-assembled nanogel & RNA enzyme delivery internalized in cells \\
\hline Poly(ethyleneimine) nanogels & $\begin{array}{l}\text { Size-dependent property } \\
\text { nanogel }\end{array}$ & $\begin{array}{c}\text { Suicide gene hTERT -CD-TK delivered for } \\
\text { lung cancer }\end{array}$ \\
\hline $\begin{array}{l}\text { Poly( } \mathrm{N} \text {-isopropylacrylamide) } \\
\text { and chitosan }\end{array}$ & $\begin{array}{l}\text { Thermosensitive } \\
\text { magnetically modalised }\end{array}$ & $\begin{array}{l}\text { Hyperthermia cancer treatment and } \\
\text { targeted drug delivery }\end{array}$ \\
\hline Poly(acrylamide) & $\begin{array}{l}\text { Novel core shell magnetic } \\
\text { nanogel }\end{array}$ & $\begin{array}{l}\text { Radiopharmaceutical carrier for cancer } \\
\text { radiotherapy }\end{array}$ \\
\hline $\begin{array}{l}\text { Methylacrylic acid and N,N'- } \\
\text { methylene-bis-(acrylamide) }\end{array}$ & $\begin{array}{c}\text { Supermagnetic nanogel } \\
\text { functionalised with carboxyl } \\
\text { group }\end{array}$ & $\begin{array}{c}\alpha \text {-chymotrypsin immobilized on aminated } \\
\text { nanogel }\end{array}$ \\
\hline $\begin{array}{l}\text { Methylacrylic acid and N,N'- } \\
\text { methylene-bis-(acrylamide) }\end{array}$ & $\begin{array}{l}\text { Magnetic nanogel } \\
\text { hydrophilic polymers }\end{array}$ & $\begin{array}{l}\alpha \text {-chymotrypsin immobilized on carboxyl } \\
\text { group }\end{array}$ \\
\hline Poly(ethyleneimine) nanogels & $\begin{array}{l}\text { Size-dependent property } \\
\text { nanogel }\end{array}$ & $\begin{array}{c}\text { Suicide gene hTERT -CD-TK delivered for } \\
\text { lung cancer }\end{array}$ \\
\hline $\begin{array}{l}\text { Acylate group modified } \\
\text { cholesterol bearing pullulan }\end{array}$ & $\begin{array}{l}\text { Cross-linked raspberry-like } \\
\text { assembly nanogel }\end{array}$ & $\begin{array}{l}\text { Efficient interleukin-12 encapsulation and } \\
\text { plasma levels }\end{array}$ \\
\hline $\begin{array}{l}\text { Poly(N-isopropylacrylamide-co- } \\
\text { acrylamide) }\end{array}$ & $\begin{array}{l}\text { In situ gelatinised thermo- } \\
\text { sensitive nanogel }\end{array}$ & $\begin{array}{l}\text { Drug loading capacity, bovine serum } \\
\text { albumin }\end{array}$ \\
\hline $\begin{array}{l}\text { Glycol chitosan grafted with 3- } \\
\text { diethylaminopropyl groups }\end{array}$ & pH-responsive & Doxorubicin uptake accelerated \\
\hline Acetylated hylauronic acid & Specific targeting nanogel & Doxorubicin loaded nanogel \\
\hline Pluronic poly(ethyleneimine) & $\begin{array}{l}\text { Temperature responsive and } \\
\text { volume transition nanogels }\end{array}$ & $\begin{array}{c}\text { Thermo responsive endosomal rupture by } \\
\text { nanogel and drug release }\end{array}$ \\
\hline
\end{tabular}

\section{Methods used for the preparation of nanogels}

I) Preparation of nanogel using polymeric precursors

II) Synthesis of nanogel network by heterogeneous polymerisation of monomers

Polymers and monomers having different nanoscopic structures formed by amphiphilic copolymers are used for the preparation of nanogels. Amphiphilic copolymers are able to self-assemble in solution, hence 
they form nanogels [24-25].

\section{I) Preparation of nanogels from polymeric precursors}

Disulphide-based cross-linking

Disulphide linkages were prepared by inverse mini-emulsion atom transfer radical polymerisation (ATRP). In this process, water-soluble monomer oligo(ethylene glycol) monomethyl ether methacrylate (OEOMA) with different molecular weights were cross-linked in the ATRP reaction with the disulphidefunctionalised cross-linker. The nanogels formed are considered to have a uniformly cross-linked network, which is supposed to improve aqueous solubility and control the release of encapsulated agents; the nanogels are shown to be biodegradable into water-soluble polymers in the presence of a biocompatible glutathione tripeptide which is commonly found in cells. The biodegradation of nanogels could trigger the release of drugs from the nanogels. Amphiphilic random copolymers are used to prepare a nanogel system by self-cross-linking [26-27]. A nanosized product in aqueous solution is formed by hydrophilic poly(ethylene glycol) and pyridyl disulphide. Thiol disulfide exchange reaction is depending primarily on the concentration of thiol exchangers like dithiothreitol (DTT). The size of the nanogel would be reduced by using cross-linking monomer or polymer chains. A lower critical solution temperature (LCST) of polymers also affects the size of the nanogel. Lipoic acid-encapsulated dextran was prepared by thiol-exchange using the same method. A catalytic amount of DTT freely cross-linked with doxorubicin was synthesised from the assembly of the polymer [28-29].

Poly( $\varepsilon$-caprolactone) (PCL) and hydrophilic poly(ethylethylenephosphate) (PEEP) were used as drug carriers for the development of a micellar nanoparticle system for intracellular drug release which is triggered by glutathione in tumour cells. Tang et al. synthesised a disulphide-linked di-block copolymer of PCL and PEEP (PCL-SS-PEEP), which forms biocompatible micelles in aqueous solution and detaches the shell material under glutathione stimulus, resulting in rapid drug release with the destruction of the micellar structure shown in Figure 1 [30].

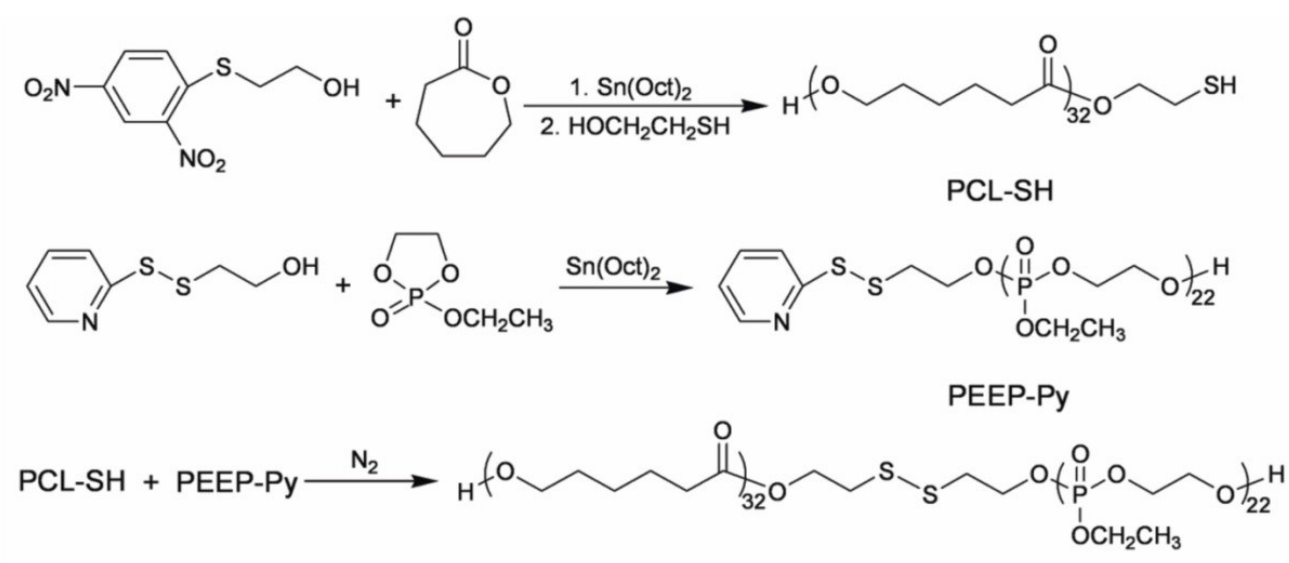

PCL-SS-PEEP

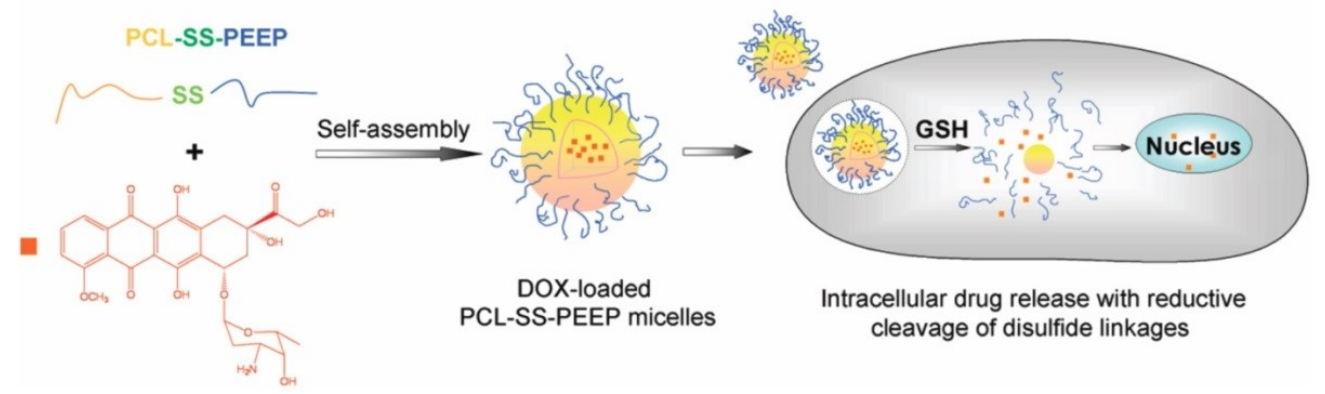

Figure 1. Synthesis pathway of the disulphide-linked PCL-SS-PEEP and schematic illustration of intracellular drug release (reprinted with the permission from [30]. Copyright (2016) American Chemical Society). 
Amine group is more common in amine-based cross-linked nanogel development because the amine group has higher reactivity against carboxylic acids, isocyanates, and iodides. Cross-linked knedelresembling structures using amine cross-linkers were prepared by the Wooley group. Hydrophilic, amphiphilic di-block copolymers were prepared by reversible addition fragmentation chain transfer polymerisation. Amidation of carboxyl group-containing self-assembled block copolymers with a diamine cross-linking agent leads to the cross-linking of micellar assemblies; the remaining carboxylic group was altered for orthogonal surface modifications in the form of other functional moieties to form a cross-linked nanogel. Furthermore, reaction with isocyanate carriers is an alternative cross-linking approach to prepare nanogels. $\mathrm{pH}$-responsive cross-linked micelles were prepared by the addition of 1,8-diaminooctane to a micellar combination of 3-isopropenyl- $\alpha, \alpha$-dimethyl benzyl isocyanate bearing copolymers [31-32].

We prepared poly(acrylic acid) (PAA) and sodium carboxymethylcellulose (NaCMC)-based luliconazole loaded nanogels. Luliconazole was encapsulated in PAA and $\mathrm{Na}-\mathrm{CMC}$ by free radical polymerisation. Luliconazole is an azole antifungal that works by preventing the growth of the fungus [33] and is used to treat skin infections such as athlete's foot, jock itch, and ringworm. Figure 2 shows particle size analysis for the optimised nanogel containing luliconazole. The average particle size of nanogel was $259 \mathrm{~nm}$ at the count rate of $360 \mathrm{kcps}$ with polydispersity index (PDI) of 0.2 showed narrow particle size distribution.

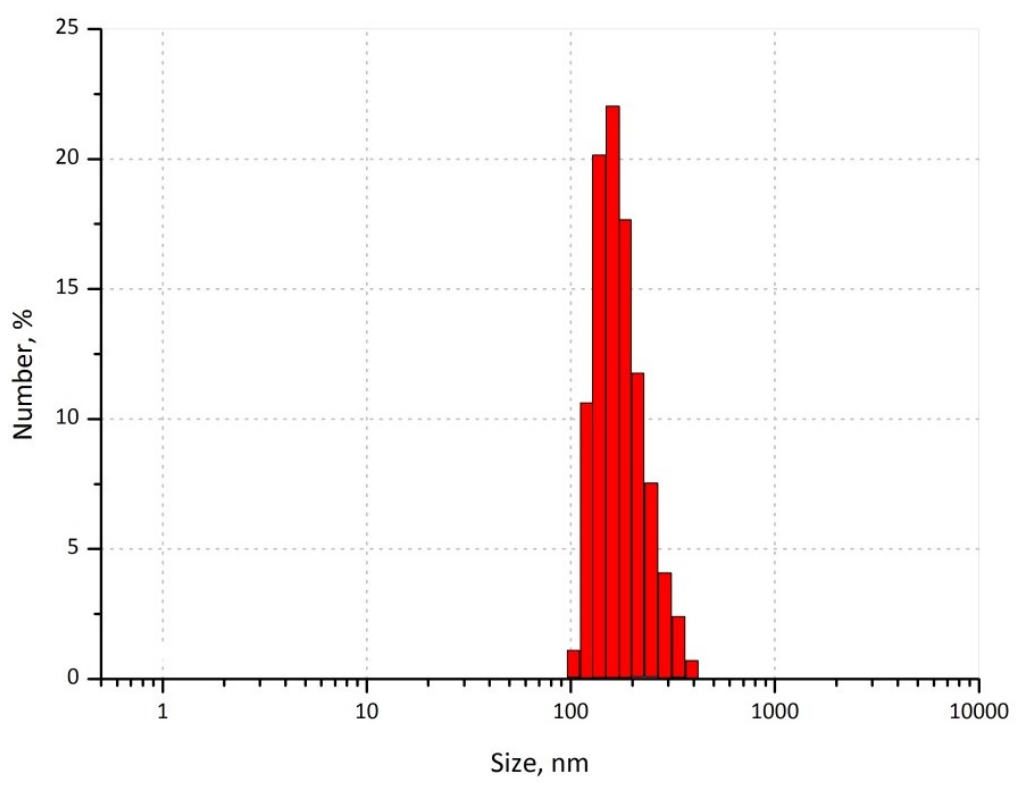

Figure 2. Average particle size of luliconazole loaded poly(acrylic acid) nanogel

The formation of nanogel (NaCMC-g-PAA) from NaCMC and acrylic acid/sodium acrylate in the presence of $\mathrm{N}, \mathrm{N}^{\prime}$-methylene bisacrylamide (MBA) was carried out using potassium persulphate as a free radical initiator [34-35]. Figure 3 shows the FESEM micrograph of the optimised nanogel formulation. From the figure, it can be observed that the formed nanogel is spherical in nature.

\section{Click chemistry-based cross-linking}

This method is discovered by Wooley and Hawker group for the synthesis of nanogels [36]. In this method, alkynyl groups were restrained to the corona of assembled micelles prepared from amphiphilic diblock copolymers of poly(acrylic acid)-b-polystyrene via the amidation of acrylic acid groups. Azido dendrimers and micelles are generally prepared by click reactions. Covalently cross-linked micelles are entrapped into the nanogel assemblies. Cross-linked polyion nanogel micelles were prepared by Liu et al. using a click chemistry approach. Core cross-linked polyion complex micelles had thermosensitive coronas with high stability against salt and $\mathrm{pH}$ [36-37]. 


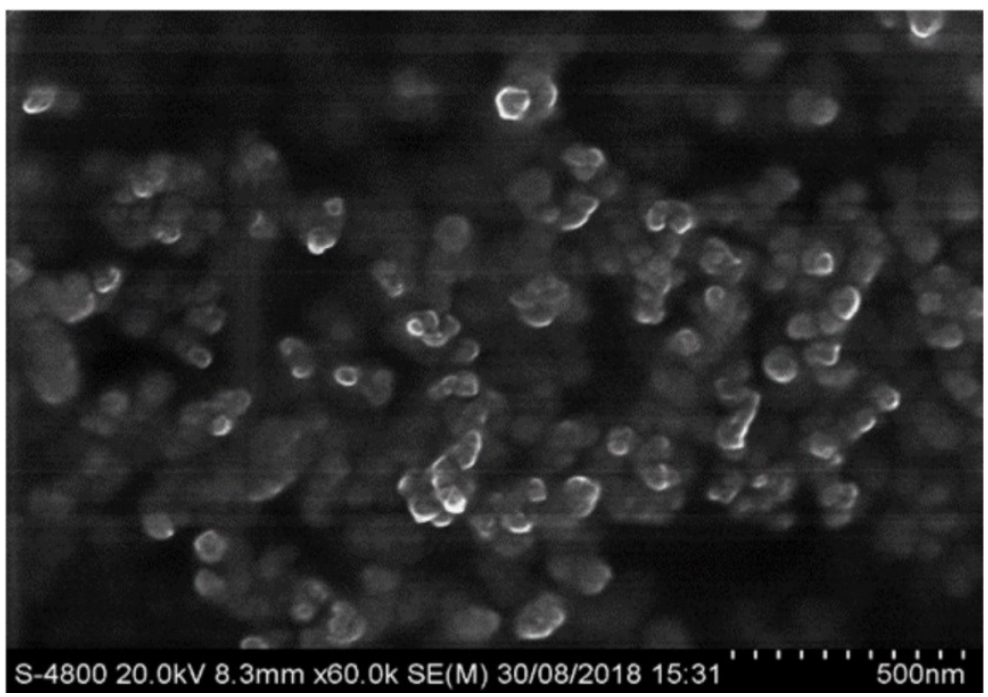

Figure 3. FESEM image of luliconazole loaded poly(acrylic acid) nanogel

Thermoresponsive poly( $\mathrm{N}$-isopropylacrylamide) (PNIPAM) represents an attractive candidate to introduce physical cross-linking via the association of hydrophobic domains because it has a gelling temperature below body temperature and good biocompatibility. PNIPAM is a non-biodegradable, thermoreversible hyaluronan-poly (N-isopropylacrylamide) (HA-PNIPAM) hydrogel with a well-defined molecular architecture and properties; this hydrogel can be synthesised through reversible addition fragmentation chain transfer (RAFT) polymerisation and "click" chemistry polymerisation method [38].

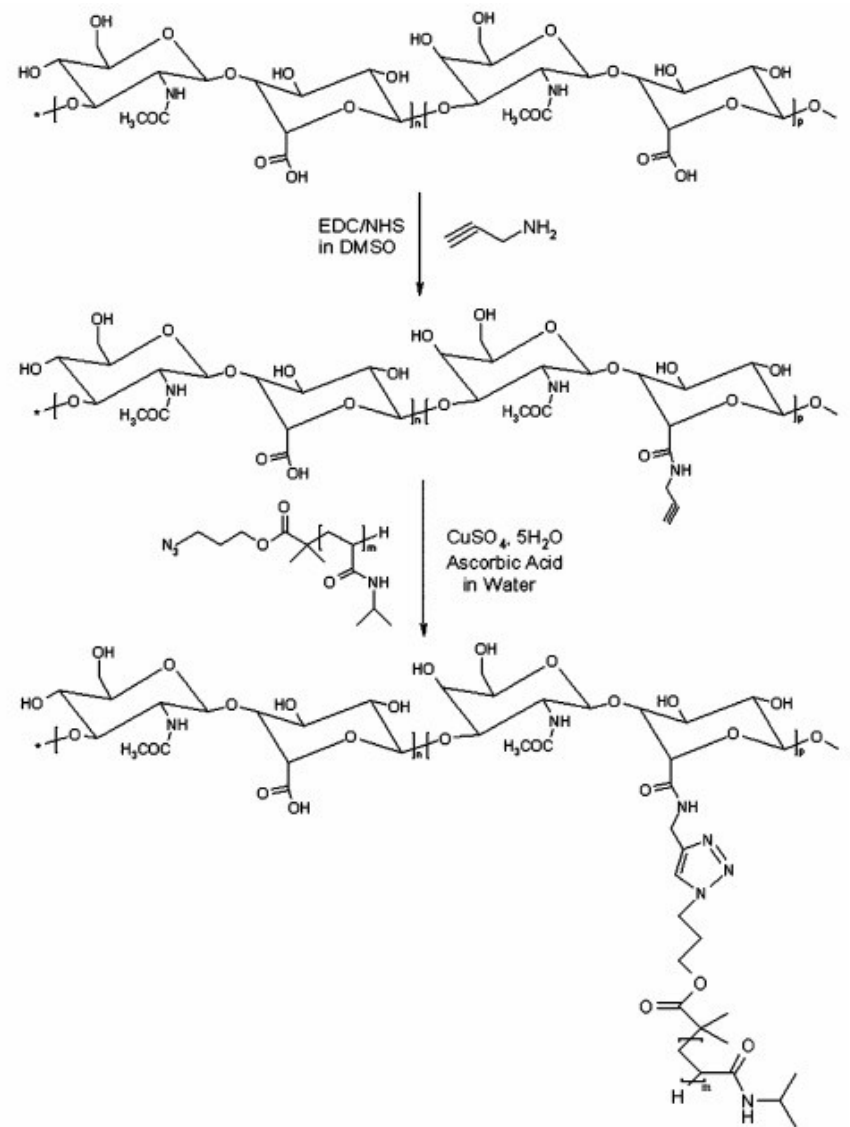

Figure 4. N-(3-dimethylaminopropyl)-N-ethylcarbodiimide hydrochloride (EDC), N-hydroxy-succinimide (NHS) synthesis of hyaluronanpropargylamide (hapa), followed by copper-catalysed azidealkyne cycloaddition of hapa with azido-terminated poly(N-isopropylacrylamide) N3-PNIPAM (reprinted with the permission from [38]. Copyright (2010) American Chemical Society).

The effect of PNIPAM grafting length and density on HA-PNIPAM properties was evaluated by methods relevant for cell therapy. It was found that the reversibility of the PNIPAM gelling process was improved in the presence of HA. The efficiency of the "click" reaction facilitates the control of the DS of PNIPAM chains. RAFT polymerisation allows the preparation of PNIPAM of controlled molecular weight and low PDI. This control of the critical parameters of PNIPAM molecular weight and grafting density allowed the gel to be 
optimised for regenerative medicinal applications. The two synthetic steps of HA-PNIPAM are shown in Figure 4. The EDC/NHS mediated coupling of PPA to carboxylic acid groups on the hyaluronan salt and the copper-catalysed azide-alkyne cycloaddition of the N3-PNIPAM to the hyaluronan-propargylamide [38-40].

\section{Photo-induced cross-linking}

This is an alternative method for nanogel preparations. In this method, the polymer chain is stabilised using cross-linking and is functionalised with dimerisable or polymerisable units. Double hydrophilic block copolymers can be encapsulated into the coumarin dimer. Coumarin dimers, when cured with UV light $>310 \mathrm{~nm}$, are assembled into micelles and then photo-cross-linked to form nanogels (Figure 5).

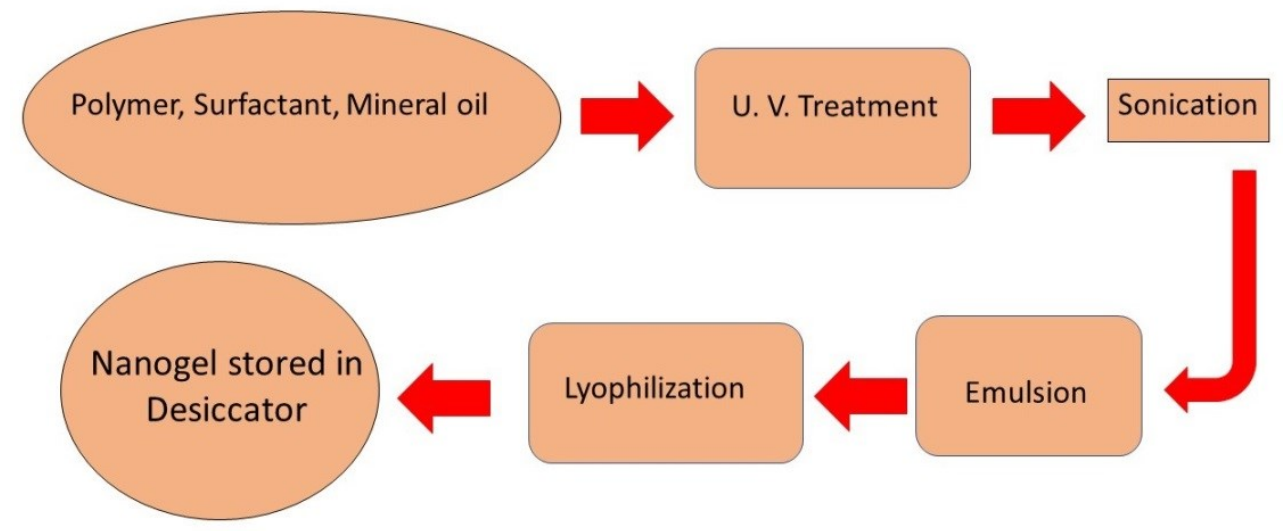

Figure 5. Scheme for UV photopolymerisation

This nanogel shows interaction between lower critical solution temperature (LCST) and upper critical solution temperature (UCST) behaviours. UCST is the critical temperature above which the contents of a mixture are miscible. The light penetrating capability has been incorporated into dendrimer structures to increase drug release in response to light stimulation. Dendrimers of coumarin act as alternative crosslinkers to control the accessibility of substrate in the nanogel networks. When the solution of coumarin was cured in UV light, the ester groups were confined in the interior assembly of nanogel. Enzymatic degradation of the substrate was very much inhibited. Curing the cross-linked assembly by UV light improves the enzymatic action due to the de-cross-linking of the coumarin dimer, which exposes the substrate to enzymes [41-43]. A stimuli-responsive nanogel prepared in water by a core cross-linking technique using diblock copolymer micelles by photo-cross-linking of the micelle core [44]. The preparation of poly(ethylene glycol)-b-poly(2-(diethylamino) ethyl methacrylate-co-2-cinnamoyloxyethyl acrylate) (PEGb-P(DEAEMA/CEA)), a pH-responsive block copolymer, by reversible addition-fragmentation chain transfer (RAFT)-controlled radical polymerisation using poly(ethylene glycol)-based chain transfer agent (PEG-CTA). The poly(ethylene glycol) (PEG) block in PEG-b-P(DEAEMA/CEA) is soluble in water, independent of $\mathrm{pH}$, while the solubility of DEAEMA depends on $\mathrm{pH}$ (Figure 6) [44-45].

We have formulated poly(acrylamide) (PAA) nanogel using UV polymerisation. PAA gels are mainly polymerised using catalysts such as tetramethylenediamine (TEMED) and ammonium persulphate (APS), which are highly toxic and lead to slow polymerisation, which is time-consuming and takes $45 \mathrm{~min}$ to $1 \mathrm{~h}$ for lower gel precursor concentrations. Photo-crosslinking with various photoinitiators, such as Irgacure 2959, has been more recently used for the synthesis of PAA hydrogels with a stiffness gradient and used for the quick preparation of large PAA hydrogel arrays for applications such as drug screening. Photo-crosslinking circumvent the use of toxic catalysts and is characteristically much faster than chemical cross-linking method. Final properties of UV-polymerized gels are depending on the UV wavelength, consistency, light intensity, and interaction time [46]. Figure 7 shows the average particle size of thymol loaded PAA nanogel and Figure 8 shows the FESEM micrograph of thymol loaded PAA. It is clear from the figure that the nanogel 
prepared by UV-polymerisation is spherical in nature.

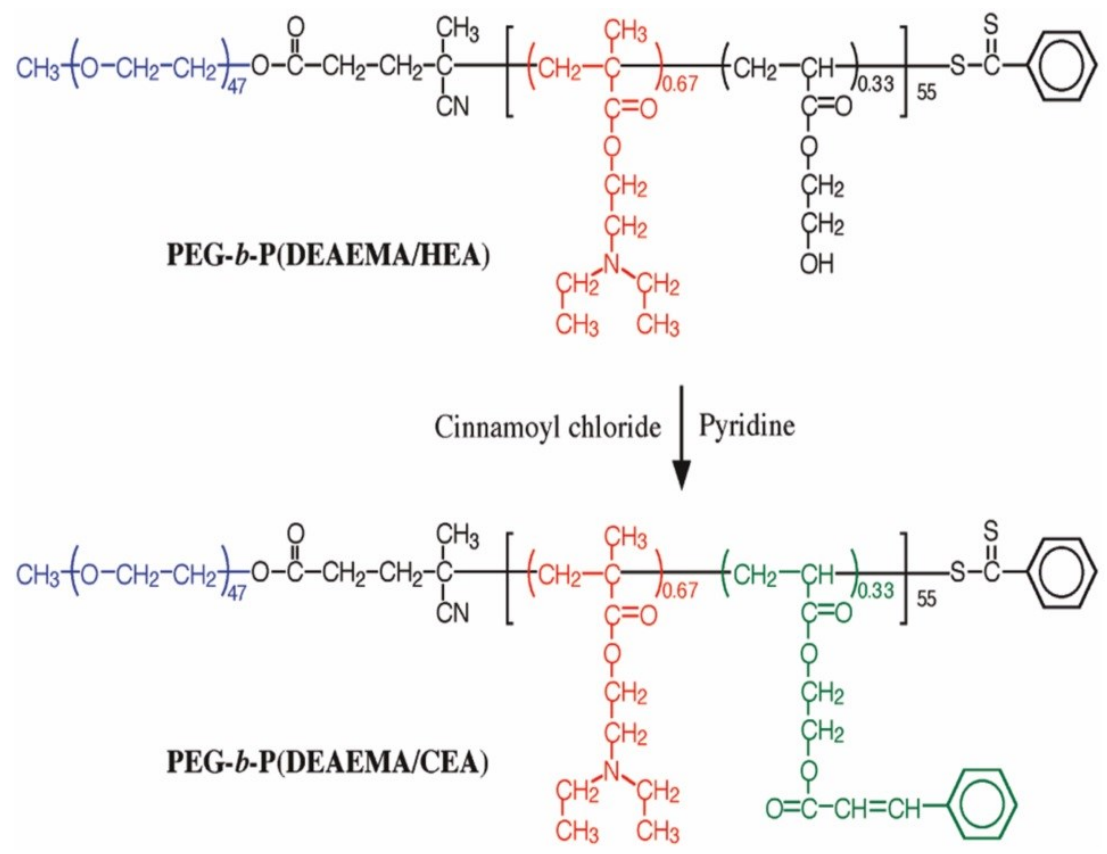

Figure 6. Synthetic route for poly (ethylene glycol)-b-poly(2-(diethylamino) ethyl methacrylate-co-2cinnamoyloxyethyl acrylate) (reprinted with permission from [45]. Copyright (2009) American Chemical Society).

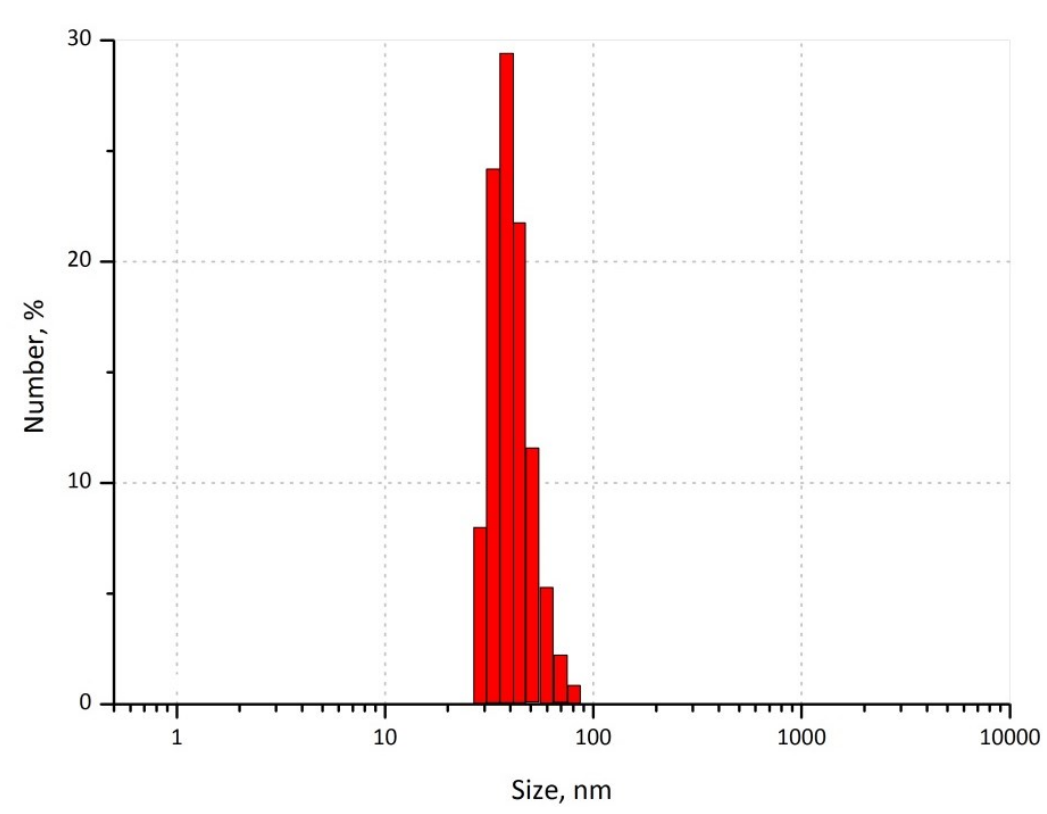

Figure 7. Average particle size of thymol loaded polyacrylamide nanogel prepared by photopolymerisation

\section{Physical cross-linking}

Physically cross-linked gels are also known as pseudo gels which have weaker van der Waals linkages, hydrogen bonding, hydrophobic or electrostatic interactions that are involved in the synthesis of pseudo gels. The physicochemical properties of gels depend on properties of the polymer, temperature, ionic strength, concentration of polymer and the cross-linking agent. Combination of amphiphilic block copolymers and complexation of oppositely charged polymeric chains is used for the formulation of pseudo gels $[43,47]$. 


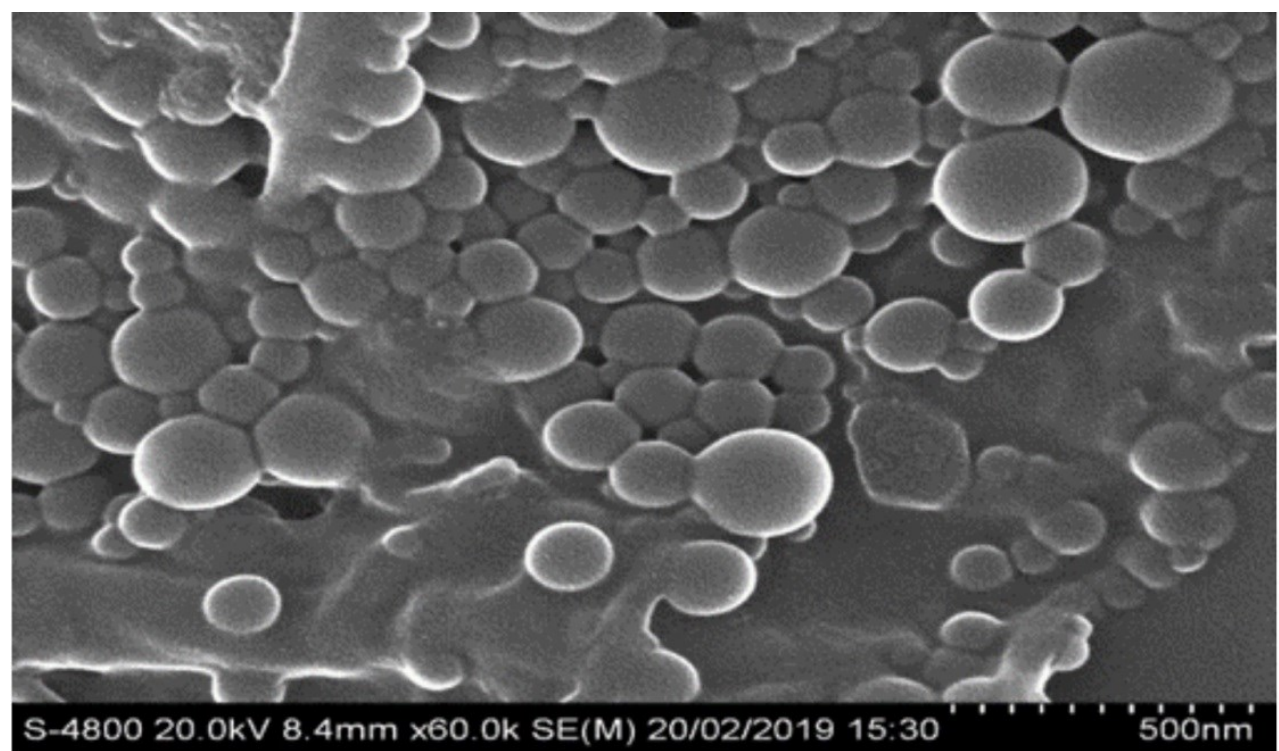

Figure 8. FESEM image of thymol loaded poly(acrylamide) acid nanogel prepared by photopolymerisation

\section{II) Synthesis of nanogel networks by heterogeneous polymerisation of monomers}

Bi-functional monomers are chemically entrapped into nanogels. Heterogeneous colloidal systems are responsible for the activation of polymerisation. Emulsion polymerisation and ATRP are used for the preparation of biodegradable nanogel. Disulphide-linked bi-functional monomers are used in the stimulation of polymerisation. Protein nanogel hybrids using ATRP in water/oil mini emulsions or inverse mini-emulsion are useful for the entrapment of covalently bonded proteins into nanogel. In the inverse mini-emulsion, a co-initiator was used to initiate the polymerisation of monomers which are firmly dispersed in the system [48-49].

\section{Drug release mechanism of nanogel}

\section{pH-responsive mechanism}

$\mathrm{pH}$-responsive, nanosized nanogels have received significant attention because of their biological relevance and due to their potential applications in drug delivery systems. Drug release is affected by the different $\mathrm{pH}$ values throughout the human body physiological conditions. $\mathrm{pH}$-responsive block copolymer micelles are suitable for controlled delivery applications. In such applications, however, the polymer micelles may experience dilutions below the critical micelle concentration $(\mathrm{cmc})$, leading to dissociation into monomers. In contrast, nanogels with a cross-linked structure are robust at a diluted concentration. Insoluble 3D structures and staying alive at low pH are the main characteristics of methacrylic acid ethyl acrylate. The polymeric chain repulsions begin and lead to the precise release profile in procaine hydrochloride due to the cumulative $\mathrm{pH}$ ranges of acidic group ionisation. Suitable $\mathrm{pH}$ at the site of action helps with the diffusion of nanogels. $\mathrm{pH}$-responsive monomers play an important role in the preparation of nanogels; these are commonly $\mathrm{pH}$-responsive functional groups that deionise in the polymeric assemblies. A nanogel containing platinum nanoparticles exhibited on and off catalytic activity for shifting reactive oxygen types. In the acidic $\mathrm{pH}$ of skin, the protonation of pendant amine of cross-linked poly $(2-(\mathrm{N}, \mathrm{N}$ diethylamino) methacrylate) core as well as PEG group in the polymer greatly enhances the solubilisation of drug [43,50-51]. 


\section{Thermosensitive and volume transition mechanism}

Variations in the capacity of nanogels according to temperature are known as the volume phase transition temperature (VPTT). Polymers become quenched and hydrated when the surrounding medium is below the VPTT. A shrunken and hydrated polymer swells and releases the loaded therapeutic agent. Thermo-responsive nanogels rupture in cells and the biological environment when they swell and rise in volume. $\mathrm{N}$-isopropyl acrylamide synthesised nanogels have thermoresponsive properties. These nanogels have important characteristics, such as rapid contraction in gel volume and the efflux of indomethacin due to the maintenance of heat beyond the lower critical solution temperature (LCST). The poly(Nisopropylacrylamide-co-acrylamide)-loaded 5-fluorouracil gel has been tested on rats in ex vivo studies. The loading of the therapeutic agent at lower temperatures and the release from nanogels at body temperature makes this suitable for drug delivery. Pluronic acid-modified thermoresponsive poly(ethyleneimine) nanogels were effectively used as gene delivery systems. Thermoresponsive nanogels with PNIPAM have very exciting and promising applications in the biomedical field, such as the treatment of certain cancers through hyperthermia. They can be loaded with an anticancer drug and, at the target location, by moderately increasing the temperature above the LCST, the nanogel can change with volume and the drug release can be increased [52].

\section{Photoisomerisation and photochemical internalization}

Stimulation of photosensitiser-loaded nanogels leads to the synthesis of singlet oxygen and reactive oxygen species which causes oxidation of cellular compartment walls such as endosomal barrier walls; this affects the release of therapeutics into the cytoplasm. An azo dextran nanogel loaded with aspirin showed the e-configuration of the azole group rather than the z-configuration at $365 \mathrm{~nm}$; cis-trans isomerisation of azobenzene by photo-regulation in an azo-dextran nanogel loaded with aspirin as a model drug exhibited that the e-configuration of the azo group leads to a better release profile of the drug than the $z$ configuration at $365 \mathrm{~nm}$ radiation $[12,43,53]$.

\section{Miscellaneous examples}

Degradation of disulphide linkages in cross-linked hyaluronic acid nanogels causes the degradation of the nanogel assembly due to the action of reducing agents; in this way, doxorubicin is released by the simple diffusive process. The size of the nanogel increases and the layer by layer release of an active ingredient is possible without a rapid burst of the drug. The release can be sustained by simple diffusion and controlled following initial release mediated by a coating with anionic and cationic polyelectrolytes [54].

\section{The application of nanogels}

Nanogel-based drug delivery formulations improve the effectiveness and safety of anti-cancer drugs, antifungal drugs, and anti-diabetic drugs, due to their physicochemical properties, as well as improving the ease of administration, as confirmed by in vivo studies. Nanogels have minimum toxicity to nearby tissues and high healing effects in cancer treatment at the site of action [55].

\section{Transdermal drug delivery of an antipyretic drug}

The nanosized dispersion of aceclofenac was formulated by emulsion-solvent diffusion methods and then incorporated into a Carbopol 940. The formulation showed optimal permeability properties and stability, and achieved a sustained drug release. A nanogel formulation containing diclofenac sodium was prepared by the emulsion-solvent diffusion method and then incorporated into a Carbopol 940 [56]. 


\section{Ophthalmic applications}

Curcumin-loaded cationic nanostructured lipid carriers (CNLC) were prepared by film-ultrasonic techniques and thermosensitive gelling agents were used to improve pre-ocular retention and the ocular permeation capacity of curcumin. Muscone has maximum drug loading in the hydrogel, and the rheology results showed that the phase transition temperature was $34^{\circ} \mathrm{C}$. Blinking of eyes was resisted due to the thixotropy; the recovery time indicated that hydrogel was effective $[43,57]$.

\section{Diabetic applications}

In diabetic patients, insulin is injected into muscles every day, which is a very painful process. To overcome this problem Lee et al. (2012) developed a chitosan-loaded inhalable deoxycholic acid altered glycol chitosan (DOCA-GC) nanogel. Nanogels are self-assembled due to hydrophobic attractions with deoxycholic acid; these nanogels formed constant hypoglycaemia over a period of 2 days comparatively at the low dose [58].

\section{Carrier for antifungal agents}

In fungal infections, physicians and patients prefer the transdermal route. A fluconazole-chitin nanogel was formulated by using regeneration chemistry and the wet milling method. Chitin nanogels were redeveloped from chitin solution. Fluconazole-chitin has a controlled release pattern which is perfect for the continuous availability of fluconazole over a longer period for effective fungal treatment [59]. The synthesis of a vitamin E nanoemulsion-based nanogel consisting of the high molecular weight active agent amphotericin $B$ has been effectively used for cutaneous fungal infections; the nanogel showed a nearly 4fold higher skin deposition through porcine ear skin [60].

\section{Nanogels in diagnostics and imaging}

Nanogels have properties like structural flexibility, high water content, fluid-like transport, biodegradability, and biocompatibility. Gadolinium-assembled nanogels were synthesised by the crosslinking of branched polyethyleneimines with gadolinium ions. Inverse microemulsion followed by surfacefunctionalisation with polyethylene glycol chains was performed to increase the blood circulation time [61].

\section{Properties of nanogels}

High water content/swellability

Nanogels have rapid swelling and de-swelling properties. Water-soluble nanogels show the benefits of hydrogels with certain advantages that are necessary for their nanoscale size. Like microgels, nanogels can contain and protect drugs and regulate their release by integrating high-affinity functional groups containing polymers $[43,62]$.

\section{Softness}

The softness of nanogels is a very important parameter in the biomedical field and alters their biodistribution properties. Softness can be adjusted by changing the chemical structure of the nanogel [63].

\section{Colloidal stability}

The surface charge of polymers inhibits the development of aggregates in the bloodstream, along with their associated problems. This can be altered by increasing the zeta potential that results in higher repulsive forces between particles which electrostatically stabilise nanogels. Another method includes the integration of surfactants like polyethylene glycol which produce a steric effect and hydration forces to give a stable nanosuspension $[43,64]$. 


\section{Biocompatibility and degradability}

Natural or synthetic polymers are used to synthesise nanogels. These are biocompatible and biodegradable, thereby preventing their accumulation in the systemic circulation. Chitosan, poly-acrylic acid, methyl cellulose, sodium alginate, and several polysaccharide-based polymers like dextran, pullulan, and cyclodextrin can be used to formulate nanogels. Polysaccharides are typically carbohydrate-based polymers formed of repeating monosaccharide units linked by glycosidic bonds. These polymers are stable, non-toxic, hydrophilic and biodegradable in nature [65].

\section{Particle size}

Nanogels are able to diffuse through the skin, tissues or compromised areas of the endothelium and in some cases through a specific transport system. Some routes of administration face the challenge of crossing the blood-brain barrier (BBB) due to their particle size. So, to overcome this issue, nanogels were developed which have a size in the diameter range from 20-200 nm. Nanogels have smaller sizes, so cross the BBB while inhibiting rapid clearance mechanisms at the same time $[43,66]$.

\section{Concluding Remarks}

Nanogels are advanced pharmaceutical nanocarriers for pharmaceutical agents as well as therapeutic agents. Nanogel systems could be easily prepared with biomacromolecules with the maximum entrapment ability and stability of the resulting formulation in dispersion. Nanogel systems control pharmaceuticallyactive compounds with different drug structures. Biopolymers and low molecular mass hydrophobes can also be encapsulated in nanogels. The discovery of a new polymeric system is very important for the development of nanogels. Advanced polymerisation or cross-linking approaches have a promising role in therapies. This is a new approach in the synthesis of nanogel assemblies. Hence, we can expect that these advanced nanocarrier systems will be focused upon in future pharmaceutical developments.

\section{Acknowledgments}

The authors would like to thank Technical Education Quality Improvement Program (TEQIP-III), World Bank and MHRD, New Delhi for providing financial support to carry out this work.

Conflicts of interest: The authors have no conflicts of interest.

\section{References}

[1] J.K. Oh, D.I. Lee, J.M. Park. Biopolymer-based microgels/nanogels for drug delivery applications. Progress in Polymer Science 34(12) (2009) 1261-1282.

[2] J.K. Oh, R. Drumright, D.J. Siegwart, K. Matyjaszewski. The development of microgels/nanogels for drug delivery applications. Progress in Polymer Science 33(4) (2008) 448-477.

[3] R.T. Chacko, J. Ventura, J. Zhuang, S. Thayumanavan. Polymer nanogels: a versatile nanoscopic drug delivery platform. Advanced Drug Delivery Reviews 64(9) (2012) 836-851.

[4] M. Hamidi, A. Azadi, P. Rafiei. Hydrogel nanoparticles in drug delivery. Advanced Drug Delivery Reviews 60(15) (2008) 1638-1649.

[5] S.A. Ferreira, F.M. Gama, M. Vilanova. Polymeric nanogels as vaccine delivery systems. Nanomedicine: Nanotechnology, Biology and Medicine 9(2) (2013) 159-173.

[6] T. Hoare, D. Sivakumaran, C.F. Stefanescu, M.W. Lawlor, D.S. Kohane. Nanogel scavengers for drugs: Local anesthetic uptake by thermoresponsive nanogels. Acta Biomaterialia 8(4) (2012) 1450-1458. 
[7] H. Ayame, N. Morimoto, K. Akiyoshi. Self-assembled cationic nanogels for intracellular protein delivery. Bioconjugate Chemistry 19(4) (2008) 882-890.

[8] J. Ramos, A. Imaz, J. Forcada. Temperature-sensitive nanogels: poly(N-vinylcaprolactam) versus poly(N-isopropylacrylamide). Polymer Chemistry 3(4) (2012) 852-856.

[9] W. Lv, S. Liu, W. Feng, J. Qi, G. Zhang, F. Zhang, X. Fan. Temperature-and Redox-Directed Multiple Self Assembly of Poly (N-Isopropylacrylamide) Grafted Dextran Nanogels. Macromolecular Rapid Communications 32(14) (2011) 1101-1107.

[10] F. Cavalieri, A. Postma, L. Lee, F. Caruso. Assembly and Functionalization of DNA- Polymer Microcapsules. Acs Nano 3(1) (2009) 234-240.

[11] M. Molinos, V. Carvalho, D.M. Silva, F.M. Gama. Development of a hybrid dextrin hydrogel encapsulating dextrin nanogel as protein delivery system. Biomacromolecules 13(2) (2012) 517-527.

[12] F. Schmitt, L. Lagopoulos, P. Käuper, N. Rossi, N. Busso, J. Barge, G. Wagnières, C. Laue, C. Wandrey, L. Juillerat-Jeanneret. Chitosan-based nanogels for selective delivery of photosensitizers to macrophages and improved retention in and therapy of articular joints. Journal of Controlled Release 144(2) (2010) 242-250.

[13] M.C. Ferrer, S. Dastgheyb, N.J. Hickok, D.M. Eckmann, R.J. Composto. Designing nanogel carriers for antibacterial applications. Acta Biomaterialia 10(5) (2014) 2105-2111.

[14] E.A. Murphy, B.K. Majeti, R. Mukthavaram, L.M. Acevedo, L.A. Barnes, D.A. Cheresh. Targeted nanogels: a versatile platform for drug delivery to tumors. Molecular Cancer Therapeutics 10(6) (2011) 972-982.

[15] S.A. Ferreira, C. Oslakovic, R. Cukalevski, B. Frohm, B. Dahlbäck, S. Linse, F.M. Gama, T. Cedervall. Biocompatibility of mannan nanogel-safe interaction with plasma proteins. Biochimica et Biophysica Acta (BBA)-General Subjects 1820(7) (2012) 1043-1051.

[16] L. He, H. Liang, L. Lin, B.R. Shah, Y. Li, Y. Chen, B. Li. Green-step assembly of low-density lipoprotein/sodium carboxymethyl cellulose nanogels for facile loading and $\mathrm{pH}$-dependent release of doxorubicin. Colloids and Surfaces B: Biointerfaces 126 (2015) 288-296.

[17] N. Li, J. Wang, X. Yang, L. Li. Novel nanogels as drug delivery systems for poorly soluble anticancer drugs. Colloids and Surfaces B: Biointerfaces 83(2) (2011) 237-244.

[18] K. Akiyoshi, N. Morimoto, H. Ayame. Self-assembled cationic nanogels for intracellular protein delivery. Bioconjugate Chemistry 19(4) (2008) 882-890.

[19] S. Honary, F. Zahir. Effect of zetz potential on the properties of nano-drug delivery systems- a review. Tropical Journal of Pharmaceutical Research 12(2) (2013) 255-264.

[20] C. Zhao, Q. Chen, K. Patel, L. Li, X. Li, Q. Wang, G. Zhang, J. Zheng. Synthesis and characterization of $\mathrm{pH}$-sensitive poly(N-2-hydroxyethyl acrylamide)-acrylic acid (poly (HEAA/AA)) nanogels with antifouling protection for controlled release. Soft Matter 8(30) (2012) 7848-7857.

[21] P. Lemieux, S. Vinogradov, C. Gebhart, N. Guerin, G. Paradis, H.K. Nguyen, B. Ochietti, Y. Suzdaltseva, E. Bartakova, T. Bronich, Y. St-Pierre. Block and graft copolymers and Nanoge ${ }^{\mathrm{TM}}$ copolymer networks for DNA delivery into cell. Journal of Drug Targeting 8(2) (2000) 91-105.

[22] Y. Lee, S.Y. Park, C. Kim, T.G. Park. Thermally triggered intracellular explosion of volume transition nanogels for necrotic cell death. Journal of Controlled Release 135(1) (2009) 89-95.

[23] Y. Sasaki, K. Akiyoshi. Nanogel engineering for new nanobiomaterials: from chaperoning engineering to biomedical applications. The Chemical Record 10(6) (2010) 366-376.

[24] Y. Xia, C. Cheng, R Wang, H. Qin, Y. Zhang, L. Ma, H. Tan, Z. Gu, C. Zhao. Surface-engineered nanogel assemblies with integrated blood compatibility, cell proliferation and antibacterial property: towards multifunctional biomedical membranes. Polymer Chemistry 5(20) (2014) 5906-5919.

[25] N. Morimoto, T. Endo, M. Ohtomi, Y. Iwasaki, K. Akiyoshi. Hybrid Nanogels with Physical and Chemical Cross-Linking Structures as Nanocarriers. Macromolecular Bioscience 5(8) (2005) 710-716. 
[26] Z.Y. Qiao, R. Zhang, F.S. Du, D.H. Liang, Z.C. Li. Multi-responsive nanogels containing motifs of ortho ester, oligo (ethylene glycol) and disulfide linkage as carriers of hydrophobic anti-cancer drugs. Journal of Controlled Release 152(1) (2011) 57-66.

[27] H. Lee, H. Mok, S. Lee, Y.K. Oh, T.G. Park. Target-specific intracellular delivery of siRNA using degradable hyaluronic acid nanogels. Journal of Controlled Release 119(2) (2007) 245-252.

[28] J. Ramos, A. Imaz, J. Forcada. Temperature-sensitive nanogels: poly(N-vinylcaprolactam) versus poly(N-isopropylacrylamide). Polymer Chemistry 3(4) (2012) 852-856.

[29] S. Patnaik, A.K. Sharma, B.S. Garg, R.P. Gandhi, K.C. Gupta. Photoregulation of drug release in azodextran nanogels. International Journal of Pharmaceutics 342(1) (2007) 184-193.

[30] L.Y. Tang, Y.C. Wang, Y. Li, J.Z. Du, J. Wang. Shell-detachable micelles based on disulfide-linked block copolymer as potential carrier for intracellular drug delivery. Bioconjugate Chemistry 20(6) (2009) 1095-1099.

[31] D. Basak, S. Ghosh. pH-Regulated Controlled Swelling and Sustained Release from the Core Functionalized Amphiphilic Block Copolymer Micelle. ACS Macro Letters 2(9) 2013 ) 799-804.

[32] O. Borisova, L. Billon, M. Zaremski, B. Grassl, Z. Bakaeva, A. Lapp, P. Stepanek, O. Borisov. Synthesis and $\mathrm{pH}$-and salinity-controlled self-assembly of novel amphiphilic block-gradient copolymers of styrene and acrylic acid. Soft Matter 8(29) (2012) 7649-7659.

[33] P. Klinpituksa, P. Kosaiyakanon. Superabsorbent polymer based on sodium carboxymethyl cellulose grafted polyacrylic acid by inverse suspension polymerization. International Journal of Polymer Science (2017) 3476921.

[34] C. Duan, D. Zhang, F. Wang, D. Zheng, L. Jia, F. Feng, Y. Liu, Y. Wang, K. Tian, F. Wang, Q. Zhang. Chitosan-g-poly(N-isopropylacrylamide) based nanogels for tumor extracellular targeting. International Journal of Pharmaceutics 409(1-2) (2011) 252-259.

[35] A. Sharma, T. Garg, A. Aman, K. Panchal, R. Sharma, S. Kumar, T. Markandeywar. Nanogel-an advanced drug delivery tool: Current and future. Artificial Cells, Nanomedicine, and Biotechnology 44(1) (2016) 165-177.

[36] R.K. Iha, K.L. Wooley, A.M. Nyström, D.J. Burke, M.J. Kade, C.J. Hawker. Applications of orthogonal "click" chemistries in the synthesis of functional soft materials. Chemical Reviews 109(11) (2009) 5620-5686.

[37] D.P. Chan, S.C. Owen, M.S. Shoichet. Double click: dual functionalized polymeric micelles with antibodies and peptides. Bioconjugate Chemistry 24(1) (2013) 105-113.

[38] D. Mortisen, M. Peroglio, M. Alini, D. Eglin. Tailoring thermoreversible hyaluronan hydrogels by "click" chemistry and RAFT polymerization for cell and drug therapy. Biomacromolecules 11(5) (2010) 1261-1272.

[39] A. Sanchez-Sanchez, I. Pérez-Baena, J. Pomposo. Advances in click chemistry for single-chain nanoparticle construction. Molecules 18(3) (2013) 3339-3355.

[40] R.T. Chacko, J. Ventura, J. Zhuang, S. Thayumanavan. Polymer nanogels: a versatile nanoscopic drug delivery platform. Advanced Drug Delivery Reviews 64(9) (2012) 836-851.

[41] J.M. Spruell, M. Wolffs, F.A. Leibfarth, B.C. Stahl, J. Heo, L.A. Connal, J. Hu, C.J. Hawker. Reactive, multifunctional polymer films through thermal cross-linking of orthogonal click groups. Journal of the American Chemical Society 133(41) (2011) 16698-16706.

[42] A. Leitner, T. Walzthoeni, R. Aebersold. Lysine-specific chemical cross-linking of protein complexes and identification of cross-linking sites using LC-MS/MS and the xQuest/xProphet software pipeline. Nature Protocols 9(1) (2014) 120-137.

[43] F. Sultana, Manirujjaman, M. Imran-UI-Haque, M. Arafat, S. Sharmin. An overview of nanogel drug delivery system. Journal of Applied Pharmaceeutical Science 3(8) (2013) S95-S105.

[44] K.W. Chun, J.B. Lee, S.H. Kim, T.G. Park. Controlled release of plasmid DNA from photo-cross-linked pluronic hydrogels. Biomaterials. 26(16) (2005) 3319-3326. 
[45] S.I. Yusa, M. Sugahara, T. Endo, Y. Morishima. Preparation and characterization of a pH-responsive nanogel based on a photo-cross-linked micelle formed from block copolymers with controlled structure. Langmuir 25(9) (2009) 5258-5265.

[46] S. Sheth, E. Jain, A. Karadaghy, S. Syed, H. Stevenson, S.P. Zustiak. UV Dose Governs UV-Polymerized Polyacrylamide Hydrogel Modulus. International Journal of Polymer Science (2017) 5147482.

[47] M.A. Pujana, L. Pérez-Álvarez, L.C. Iturbe, I. Katime. Biodegradable chitosan nanogels crosslinked with genipin. Carbohydrate Polymers 94(2) (2013) 836-842.

[48] T.G. Van Thienen, K. Raemdonck, J. Demeester, S.C. De Smedt. Protein release from biodegradable dextran nanogels. Langmuir 23(19) (2007) 9794-9801.

[49] W.C. Lee, Y.C. Li, I. Chu. Amphiphilic Poly(D,L-lactic acid)/Poly(ethylene glycol)/Poly(D,L-lactic acid) Nanogels for Controlled Release of Hydrophobic Drugs. Macromolecular Bioscience 6(10) (2006) 846854.

[50] A. Tamura, M. Oishi, Y. Nagasaki. Enhanced cytoplasmic delivery of siRNA using a stabilized polyion complex based on PEGylated nanogels with a cross-linked polyamine structure. Biomacromolecules 10(7) (2009) 1818-1827.

[51] G. Tamura, Y. Shinohara, I. Akiba, A. Tamura, M. Oishi, Y. Nagasaki, K. Sakurai, Y. Amemiya. pHresponsive structural change of PEGylated amine-bearing nanogel explored by small angle $\mathrm{X}$-ray scattering. Journal of Physics: Conference Series 272 (2011) 012018.

[52] H. Tokuyama, Y. Kato. Preparation of poly ( $\mathrm{N}$-isopropylacrylamide) emulsion gels and their drug release behaviors. Colloids and Surfaces B: Biointerfaces 67(1) (2008) 92-98.

[53] R. Cheng, F. Feng, F. Meng, C. Deng, J. Feijen, Z. Zhong. Glutathione-responsive nano-vehicles as a promising platform for targeted intracellular drug and gene delivery. Journal of Controlled Release 152 (2011) 2-12.

[54] P.P. Constantinides, M.V. Chaubal, R. Shorr. Advances in lipid nanodispersions for parenteral drug delivery and targeting. Advanced Drug Delivery Reviews 60 (2008) 757-767.

[55] H.S. Abandansari, M.R. Nabid, S.J. Rezaei, H. Niknejad. pH-sensitive nanogels based on Boltorn ${ }^{\circledR} \mathrm{H} 40$ and poly (vinylpyridine) using mini-emulsion polymerization for delivery of hydrophobic anticancer drugs. Polymer 55(16) (2014) 3579-3590.

[56] S. Talele, P. Nikam, B. Ghosh, C. Deore, A. Jaybhave, A. Jadhav. A Research Article on Nanogel as Topical Promising Drug Delivery for Diclofenac Sodium. Indian Journal of Pharmaceutical Education and Research 51 (4S) (2017) 5680-5685.

[57] R. Liu, L. Sun, S. Fang, S. Wang, J. Chen, X. Xiao, C. Liu. Thermosensitive in situ nanogel as ophthalmic delivery system of curcumin: development, characterization, in vitro permeation and in vivo pharmacokinetic studies. Pharmaceutical Development and Technology 21(5) (2016) 576-582.

[58] G. Wang, Q. Nie, C. Zang, B. Zhang, Q. Zhu, G. Luo, S. Wang. Self-assembled thermoresponsive nanogels prepared by reverse micelle $\rightarrow$ positive micelle method for ophthalmic delivery of muscone, a poorly water-soluble drug. Journal of Pharmaceutical Sciences 105(9) (2016) 2752-2759.

[59] J. Lee, C. Lee, T.H. Kim, E.S. Lee, B.S. Shin, S.C. Chi, E.S. Park, K.C. Lee, Y.S. Youn. Self-assembled glycol chitosan nanogels containing palmityl-acylated exendin-4 peptide as a long-acting anti-diabetic inhalation system." Journal of Controlled Release 161(3) (2012) 728-734.

[60] M. Nishil, S.N. Rejinold, S. Mangalathillam, R. Biswas, S. Nair, R. Jayakumar. Fluconazole loaded chitin nanogels as a topical ocular drug delivery agent for corneal fungal infections. Journal of Biomedical Nanotechnology 9(9) (2013) 1521-1531.

[61] L. Kaur, S.K. Jain, K. Singh. Vitamin E TPGS based nanogel for the skin targeting of high molecular weight anti-fungal drug: development and in vitro and in vivo assessment. RSC Advances, 5 (2015) 53671-53686.

[62] L.C. Keun, A. Singh, J. Heo, D. Kim, K.E. Lee, H. Jeon, J. Koh, I.C. Kwon, S. Kim. Gadolinium-coordinated elastic nanogels for in vivo tumor targeting and imaging. Biomaterials 34(28) (2013) 6846-6852.

[63] I. Neamtu, A.G. Rusu, A. Diaconu, L.E. Nita, A.P. Chiriac. Basic concepts and recent advances in nanogels as carriers for medical applications. Drug Delivery 24(1) (2017) 539-557. 
[64] M.D. Moya-Ortega, C. Alvarez-Lorenzo, A. Concheiro, and T. Loftsson. Cyclodextrin-based nanogels for pharmaceutical and biomedical applications. International Journal of Pharmaceutics 428(1-2) (2012) 152-163.

[65] K.S. Soni, S.S. Desale, T.K. Bronich. Nanogels: An overview of properties, biomedical applications and obstacles to clinical translation. Journal of Controlled Release 240 (2016) 109-126.

[66] H.K.S Yadav, N.A. Al Halabi, G.A. Alsalloum. Nanogels as Novel Drug Delivery Systems-A Review. Journal of Pharmacy and Pharmaceutical Research 1 (2017) 5.

C 2020 by the authors; licensee IAPC, Zagreb, Croatia. This article is an open-access article distributed under the terms and conditions of the Creative Commons Attribution license (http://creativecommons.org/licenses/by/3.0/) (cc)) BY 